\title{
A quality function deployment model of best practices in customer-supplier relationships
}

\author{
A.K.Kochhar ${ }^{1)}$, M.K.Saeed ${ }^{2)}$ \\ ${ }^{1)}$ School of Engineering and Applied Science \\ Aston University, Birmingham B4 7ET, United Kingdom \\ Tel: (+44) 1213593611 \\ Fax: (+44) 1213598482 \\ e-mail: A.K.Kochhar@aston.ac.uk \\ ${ }^{2)}$ Department of Mechanical Engineering \\ UMIST, Manchester M60 1QD, United Kingdom
}

\begin{abstract}
This paper outlines the use of networks and quality function deployment to represent the relationships between best practices, measures of performance and associated variables in customer-supplier relationships. It is shown that the effective implementation of many customer -supplier relationship best practices requires the prior implementation of a number of best practices.
\end{abstract}

\section{Keywords}

Customer-Supplier Partnerships; Best Practices; Measures of Performance; Quality Function Deployment

\section{INTRODUCTION}

Changing world competitive conditions and the demand for enhanced standards of performance with respect to quality, delivery and costs of supply have led to companies going for a long term relationship/partnership with their suppliers. Partnerships are seen as the ideal way by which philosophies of JIT and TQM can be diffused and implemented throughout the network of suppliers. Within the span of customer-supplier partnerships, implementation of best practices can lead to the improvement of various measures of performance which are oriented to customers. 
There is a need to develop cause and effect relationships among best practices, measures of performance and variables to study the impact of implementation of these practices on the top level strategic objectives.

Within this context a best practices model has been developed highlighting the fact that the partnership is a culture-based process that takes time and effort, and reaches every part of the business. This has been achieved by analyzing the impact of the found best practices on various performance indicators which are considered as top level strategic objectives. Similarly it has been found that the implementation of practices or manipulating variables may improve performance in certain areas but it might not have any affect on other areas.

\section{RELATIONSHIPS AMONG MEASURES OF PERFORMANCE, BEST PRACTICES AND VARIABLES}

Recent work at UMIST [Kennerley et al, 1996] addresses the relationship between performance and practices. The research resulted in a framework in which the top level objectives of an organisation (defined as competitive priorities in terms of cost, quality, flexibility and delivery) are linked to the performance measures for a generic Manufacturing Planning and Control System (MPCS). This framework suggests the use of associated best practices to stimulate performance at lower levels and as a consequence achieve improvements at top levels. It also integrates operational variables whose manipulation contributes to improved performance at all levels, and links them to best practices.

Within the context of customer-supplier partnerships the implementation of best practices to attain top level strategic objectives may result in positive or negative effects in the magnitude of certain measures of performance. This may also modify certain variables, which indirectly affect the various measures of performance. All this exposes the need to consider Cause and Effect relationships among the various elements to evaluate the impact on performance due to the implementation of various best practices.

In the context of customer-supplier relationships, the following 24 best practices (referred to as BP's) have been identified.

BP1 Developing appraisal criteria to select a partner

BP2 Selection of supplier or a partner for a long term relationship.

BP3 Working towards achieving external centres of excellence

BP4 Setting up of clear and common objectives for the defined strategic intent by both parties to minimize duplication and focus on the efforts during project execution.

BP5 Agreeing the style of relationship. 
BP6 Partners should draw strength from one another. Once the goals have been established, it should be determined what can be brought to the table.

BP7 Removal of ambiguous relationships between partners.

BP8 Management having the capacity to learn and apply the results to partnership.

$\boldsymbol{B P 9}$ Management should grasp the new situation with vigour and conviction and keep a balance between trust and self interest.

BP10 Implementing a proper structural form of collaboration to meet the strategic intent of the partners.

BP11 Looking at ways to capitalize on the Internet \& Intranets and grabbing full scope of opportunities available with customers

BP12 Establishing a level of communication with suppliers that fosters continuous improvement and problem solving

BP13 Correlation of information flow and merging of technology between the firms involved in customer-supplier partnerships

BP14 Working towards improving the quality and encouraging suppliers to achieve third party quality assurance certification to ISO9000.

BP15 Working together in improving the design process.

BP16 Working towards improving material flow thus helping towards JIT manufacturing concept

BP17 Focusing on quick and effective implementation of the results for the partner firms

BP18 Working towards cost sharing and building financial co-operation

BP19 Using Purchasing cards as a new tool to foster partnerships

$\boldsymbol{B P 2 0}$ Investing in training, learning and development

BP21 Partners investing in a marketing program which can cost little but reap good rewards

BP22 Continuously monitoring the results against the objectives by setting targets and having a criteria for judging the progress of alliance

BP23 Working towards an insourcing program

BP24 Anticipating and managing alliance dynamics over time.

The relationships between individual best practices can be represented in the form of a Network, as shown in Figure 1. This type of representation is based on the fact that no operation can exist on its own. There should be a series of operations behind it. The main purpose of using this technique is to show which immediate practices have to be implemented first to make the particular practice possible. The extent of representation of the prerequisite practices is limited to two levels, though the levels can be extended depending on the prerequisites

\section{CAUSE AND EFFECT RELATIONSHIPS}

For the representation of relationships between the best practices, variables and measures of performance, a series of matrices have been created following a 
Quality Function Deployment (QFD) framework. Application of the QFD approach for representing cause and effect relationships in customer -supplier relationships involves a series of phases to show how the implementation of various practices affects higher level objectives. This can be carried out as follows:

Measures of Performance (MOP) - Best Practices $(B P)$. This describes how the implementation of various best practices can lead to improving or worsening of various performance measures.

Measures of Performance (MOP) - Variables (V). This heading indicates how the different variables contribute to improving or worsening performance.

Best Practice (BP) - Variables (V). This shows how the implementation of various best practices can lead to different levels of improvement in the magnitudes of variables.

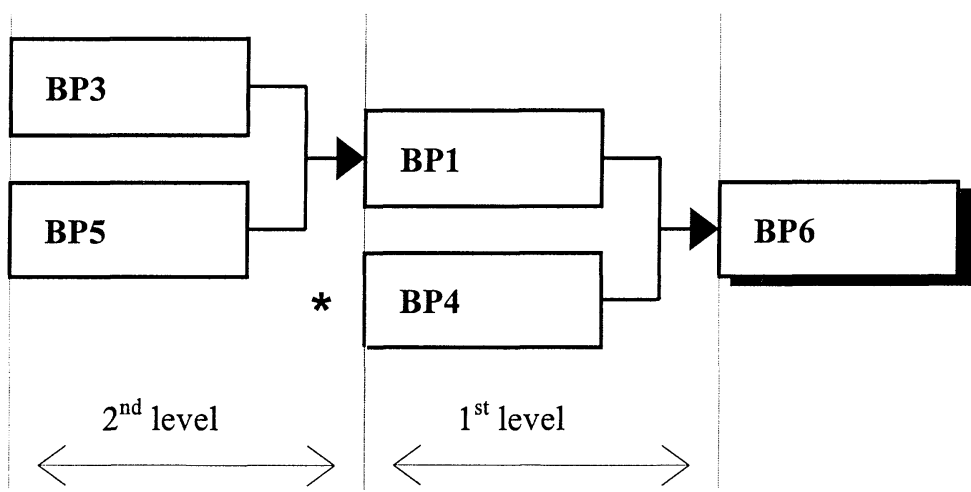

Figure 1 Network representation of relationships between best practices

\subsection{Measures of Performance - Best Practices}

The relationships between the MOPs and the best practices are illustrated by assessing the impact of Best Practice 1 (BP1) - Developing appraisal criteria to select a partner on the 5 defined measures of performance.

- Improving quality [+ Strong]

In business there is only a limited amount of time to cultivate relationships with a small number of suppliers. The supplier selected should have the technical expertise necessary along with the quality assurance certification related to the items to be supplied.

- Reducing costs [+ Strong]

The practice leads to the creation of specific data about service, quality and cost needs of each department in the company. Focus on documenting and understanding the needs and desires of the company should lead to the selection of a competent supplier resulting in the reduction of overall acquisition costs.

- Reducing product cycle time [+ Medium] 
The practice should lead to the selection of suppliers who have the technical expertise and technology to reduce the product cycle time by working with each other.

- Customer satisfaction level [+ Strong]

The practice leads to an increase in the level of customer satisfaction by enabling the management to undertake research to find suppliers who have adequate quantitative and proactive measures showing how well they are satisfying their customer needs and expectations.

- On time delivery performance [+ Medium]

One criterion to consider during the selection of a partner would be its past delivery performance. The practice should ensure that only those suppliers are selected who can deliver the products as and when they are required.

\subsection{Measures of Performance - Variables}

The relationships between the measures of performance and variables are illustrated by examining the impact of important variables on the measure of performance - improving quality.

- Level of communication [+ Strong]

According to Deming [1982] and Ishikawa [1985] non-conformance of purchased items is often due to the customer's inability to communicate the requirements clearly. The supplier must be given the opportunity to understand the function of the part being supplied and discuss design details, particularly with regard to key features and the manufacturability of purchased or subcontracted items before requirements are finalized.

- Level of focus on learning \& development [+ Strong].

Learning related to the use of multi-functional sourcing teams and simultaneous engineering approaches to product design and development, can enhance the new product development process in terms of quality, cost and time [Saunders, 1997].

- Level of teamworking [+ Strong]

Increasing the level of team work leads to Total Quality Management and improvement. It also helps in managing uncertainty, integrating tasks and information.

- Level of continuous improvement [+ Strong]

Continuous improvement suggests that the quality work is long term. By keeping an open mind to improvements, organising feedback from customers, sales people, service people and so on, and by making use of advances in technology, management techniques and so forth, quality can be continuously improved.

- Level of performance feedback to and from suppliers [+ Strong]

The implementation of new concepts, the experience gained through the implementation, feedback and revision, all done in real settings and in various types of service organisation, are necessary to prove the validity of the new quality thinking. 


\section{- Level of co-operation [+ Strong]}

Increase in level of co-operation between the partners will lead the emphasis to be placed on getting the quality right the first time, rather than on costly inspections later.

\subsection{Best Practices - Variables}

The relationships between the best practices and variables are illustrated by considering the impact of Best Practice 2 - Selection of a Supplier for a long term relationship on four variables.

- Level of trust [+ Strong]

Consolidating the supply base allows partnering which engenders trust and with trust comes openness towards a common goal [Karrass, 1997].

- Level of waste [+ Strong]

Doing business together will lead to removing duplicated effort, eliminate waste and bureaucracy in the system, and removing practices that don't add value. The ability to take out waste depends on a close relationship with the suppliers [Smith, 1998].

- Level of team working [+ Strong]

The practice should lead to the formation of joint teams which can identify improvements and ideas can be piloted, monitored and assessed with greatly reduced risk.

- Level of co-operation [+ Strong]

The practice should lead to a high level of co-operation where the customers and suppliers are in the same business and working together and there should be no loser.

Lack of space prevents a detailed discussion of all the relationships. It has been possible to provide only a glimpse of all the relationships that have been developed.

\section{VERIFICATION OF THE RELATIONSHIPS}

To verify all the concepts addressed in this investigation an industrial validation was carried out with two different companies. The aim of the industrial investigation was to assess the practical application of the developed Best Practices Model in Customer-Supplier Partnerships. A document was sent to each of the participants. The document contained seven sections which included the outline of best practices in customer-supplier partnerships, a brief description of the found measures of performance along with the variables, 2-level network representation and QFD cause and effect relationship tables along with their justification and comments. 
A two part questionnaire was also sent along with the document to seek information and comments regarding the research done. The first part of the questionnaire was designed to ask specific questions about the perception of each participant about the found practices, use of QFD to explain more easily the cause and effect concepts and 2-level network representation technique. The second part of the questionnaire was designed to determine if any of the best practices and measures of performance found during the course of this research were already being used in their respective organisations. All measures of performance were considered as very relevant for assessing the performance of customer-supplier partnerships. It was recognized that the investigated measures of performance can be used by different manufacturing firms as they cover general concepts which are important for these firms. They consider these as sufficient to judge the performance of suppliers. The variables identified in this research were considered to be adequate within the context of customer-supplier partnerships. The QFD technique employed to represent the cause and effect relationships between the best practices, measures of performance and variables was found to be appropriate. It was felt that it is very complex to analyze the visual representation of prerequisite relationships using the QFD format. The network analysis allows a more detailed picture of what is involved in implementing a practice. The industrial investigation indicated that most of the effects shown in Cause and Effect relationships tables coincide with the effects expected in practice.

\section{CONCLUSIONS}

Best practices can lead to significant benefits for partners in a long term customersupplier relationship. The implementation of best practices should go together with the establishment of the measures of performance. There are many complex relationships between the best practices, measures of performance, and associated variables. Network and Quality Function Deployment approach based models provide visual representations of these relationships. The 3 Stage QFD model, although complex, makes it possible to take account of all the cause and effect relationships. In contrast, the network representation approach is simple, easy to understand, and capable of representing the multi-level dependency relationships between best practices. However it is of limited use for representing all of the cause and effect relationships involved.

\section{REFERENCES}

Baran, John. [1992], "CIGNA Begins its Quality Transformation” Quality Progress/ January 1992

Deming, W. E. [1982]"Quality, Productivity and Competitive Position" Massachusetts Institute of Technology, Cambridge, MA. 
Ishikawa, K. [1985] "What is total quality control? The Japanese way" Prentice Hall, New Jersey.

Karrass, C. [1997] "How to handle hecklers". Subject: Negotiation perspective http://www.manufacturing.net/magazine/purchasing/.

Kennerley, M.P., Davies A. and Kochhar, A.K. [1996],"The Integration of Strategic Measures of Performance and Cause and Effect relationships in Manufacturing Planning and Control Systems" Proc. IFIP Intl. Workshop on Modeling Techniques, Business Processes and Benchmarks, University of Bordeaux, 18-19 April.

Saunders, M. [1997]“'Strategic Purchasing and Supply Chain Management” $2^{\text {nd }}$ Edit 